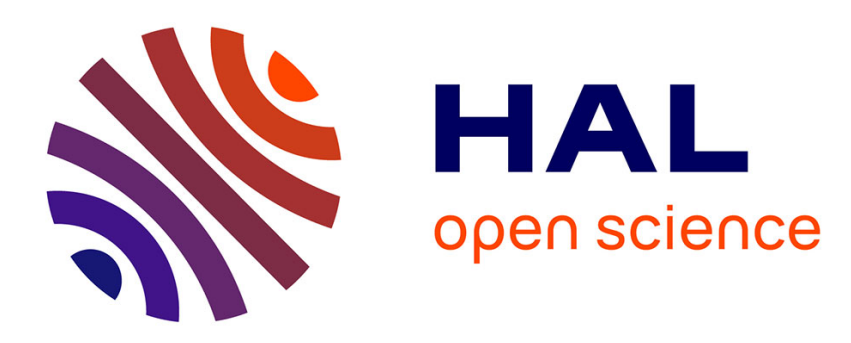

\title{
SOME RELATIONS BETWEEN BOUNDED BELOW ELLIPTIC OPERATORS AND STOCHASTIC ANALYSIS
}

Remi Leandre

\section{To cite this version:}

Remi Leandre. SOME RELATIONS BETWEEN BOUNDED BELOW ELLIPTIC OPERATORS AND STOCHASTIC ANALYSIS. Fractional Calculus., In press. hal-02367486

\section{HAL Id: hal-02367486 https://hal.science/hal-02367486}

Submitted on 18 Nov 2019

HAL is a multi-disciplinary open access archive for the deposit and dissemination of scientific research documents, whether they are published or not. The documents may come from teaching and research institutions in France or abroad, or from public or private research centers.
L'archive ouverte pluridisciplinaire HAL, est destinée au dépôt et à la diffusion de documents scientifiques de niveau recherche, publiés ou non, émanant des établissements d'enseignement et de recherche français ou étrangers, des laboratoires publics ou privés. 


\title{
SOME RELATIONS BETWEEN BOUNDED BELOW ELLIPTIC OPERATORS AND STOCHASTIC ANALYSIS
}

\author{
Rémi Léandre \\ Laboratoire de Mathématiques, \\ Université de Bourgogne-Franche-Comté, \\ 25030, Besançon, FRANCE. \\ email: remi.leandre@univ-fcomte.fr
}

April 11, 2019

\begin{abstract}
We apply Malliavin Calculus tools to the case of a bounded below elliptic rightinvariant Pseudodifferential operators on a Lie group. We give examples of bounded below pseudodifferential elliptic operators on $\mathbb{R}^{d}$ by using the theory of Poisson process and the Garding inequality. In the two cases, there is no stochastic processes besides because the considered semi-groups do not preserve positivity.

keywords:Malliavin Calculus. Pseudodifferential operators. Generalized Poisson processes. Garding inequality
\end{abstract}

\section{INTRODUCTION}

Let $G$ be a compact connected Lie group, with generic element $g$ endowed with its binvariant Riemannian structure and with its normalized Haar measure $d g$. $e$ is the unit element of $G$.

Let $f^{i}$ be a basis of $T_{e} G$. We can consider as rightinvariant vector fields. This means that if we consider the action $R_{g_{0}} h \rightarrow\left(g \rightarrow h\left(g g_{0}\right)\right)$ on smooth function $h$ on $G$, we have

$$
R_{g_{0}}\left(f^{i} h\right)=f^{i}\left(R_{g_{0}} h\right)
$$

We consider a rightinvariant elliptic pseudodifferential bounded below elliptic operator $L$ of order larger than $2 k$ on $G$. It generates by elliptic theory a semi group $P_{t}$ on $L^{2}(d g)$ and even on $C_{b}(G)$ the space of continuous functions on $G$ endowed with the uniform norm. 
Theorem 1 If $t>0$,

$$
P_{t} h\left(g_{0}\right)=\int_{G} p_{t}\left(g_{0}, g\right) h(g) d g
$$

where $g \rightarrow p_{t}\left(g_{0}, g\right)$ is smooth if $h$ is continuous.

This theorem is classical in analysis, but it enters in our general program to implement stochastic analysis tools in the theory of Non-Markovian semi-group. See the review [7] and [13] for that. See [10], [11] for another presentation.

In [12], we have considered the case of rightinvariant differential operators. The algebraic statzement of the proof is an improvement of the proof of [12], but the estimates are the same, based upon a suitable Davies gauge transform.

Unlike the Malliavin Calculus for jump processe [1], [5], [6]), there is no limitation here on the size of jumps. We give an example of bounded below pseudodifferential operator, whose origin comes from the theory of Poisson processes in the last part of this work. Unlike as it is traditional in stochastic analysis, where power between 0 and 1 of diffusion operator can be studied as jump process, we can apply this work to any positive power of a right invariant strictly positive differential operator on $G([14])$.

\section{PSEUDODIFFERENTIAL OPERATORS}

Let us recall what is a pseudodifferential operator on $\mathbb{R}^{d}([3],[5],[6]$, [15]). Let be a smooth function function from $\mathbb{R}^{d} \times \mathbb{R}^{d}$ into $\mathbb{C} a(x, \xi)$. We suppose that for all $x$

$$
\left|D_{x}^{r} D_{\xi}^{l} a(x, \xi)\right| \leq C|\xi|^{m-l}+C
$$

We suppose that for all $x$

$$
|a(x, \xi)| \geq C|\xi|^{m^{\prime}}
$$

for $|\xi|>C$ for a suitable $m^{\prime}>0$. Let $\hat{h}$ the Fourier transform of the continuous function $h$. We consider the operator $L$ defines on smooth function $h$ by:

$$
\hat{L h}(x)=\int a(x, \xi) \hat{h}(\xi) d \xi
$$

$L$ is said to be a pesudodifferential operator elliptic of order larger than $m^{\prime}$ with symbol $a$. This property is invariant if we do a diffeomorphism on $\mathbb{R}^{d}$ with bounded derivatives at each order. This remark allows to define by using charts a pseudodifferential operator elliptic of order larger than $m^{\prime}$ on a compact manifold $M$.

On a compact Riemannian manifold, we can consider the Riemannian measure. In local coordinates, the Riemannian metric is given by a smooth map

$$
x \rightarrow g_{i, j}(x)
$$

in the set of strictly positive matrix and the Riemannian measure is given by

$$
d x=\operatorname{det}\left(g_{., .}\right)^{-1 / 2} d x_{1} \otimes . . \otimes d x_{d}
$$


We can normalize the Riemannian measure to be of total mass 1.

The fact that $L$ is symmetric on $L^{2}(M)$ means that

$$
\int_{M}<h_{1}(x), L h_{2}(x)>d x=\int_{M}<L h_{1}(x), h_{2}(x)>d x
$$

The fact that $L$ is bounded below means that for some $C>0$ :

$$
\int_{M}<h(x), L h(x)>d x \geq-C \int_{M}<h(x), h(x)>d x
$$

In such a case $L$ has a self adjoint extension. This generates a semi-group of bounded operators $P_{t}$ on $L^{2}(M)$ satisfying the heat equation:

$$
\frac{\partial}{\partial t} P_{t} h=-L P_{t} h
$$

for $h \in L^{2}(M)$ and $t>0$. Moreover we suppose that $P_{0} h=h$. It generates moreover a semi-group on $C_{b}(M)$ by ellipticity.

An example can be given on $\mathbb{R}^{d}$ if we use the Garding inequality ([15]). Suppose that we consider the Lebesgue measure on $\mathbb{R}^{d}$ and that for $|\xi|>C_{0}$ we have

$$
\operatorname{Re}(a(x, \xi))>C|\xi|^{m^{\prime}}
$$

for some $C>0$. In such a case if we suppose $L$ symmetric, it is bounded below.

\section{PROOF OF THE THEOREM}

The algebraic part of this work is slighly different of [12]. We give the details and we don't write to write the details of the difficulty which comes from the fact we use no bounded functions in the enlarged semi-group whose treatment is exactly the same as in [12].

We consider the family of operators on $C^{\infty}\left(G \times \mathbb{R}^{n}\right)$ :

$$
\tilde{L}_{t}^{n}=L+\sum_{i=1}^{n} f^{j_{i}} \frac{\partial}{\partial u_{i}} \alpha_{t}^{i}+\sum_{i=1}^{n} \frac{\partial^{2 k}}{\partial u_{i}^{2 k}}
$$

$\alpha_{t}^{i}$ are smooth function from $\mathbb{R}^{+}$into $\mathbb{R}$. By elliptic theory, $\tilde{L}_{t}^{n}$ generates a semi-group $\tilde{P}_{t}^{n}$ on $C_{b}\left(G \times R^{n}\right)$. This semi-group is time inhomegeneous.

$$
\tilde{P}_{t}^{n+1}\left[h(g) h^{n}(u) v\right](., ., 0)=\int_{0}^{t} \tilde{P}_{t, s}^{n}\left[f^{j+1} \alpha_{s}^{n+1} \tilde{P}_{s}^{n}\left[h(g) h^{n}(u)\right](., .)\right.
$$

Moreover

$$
\begin{aligned}
& \tilde{P}_{t}^{n+1}\left[u h(.) h^{n}(.)\right]\left(., ., u_{n+1}\right)= \\
& \quad \tilde{P}_{t}^{n+1}\left[u h(.) h^{n}(.)\right](., ., 0)+\tilde{P}_{t}^{n}\left[h(.) h^{n}(.)\right](., .) u_{n+1}
\end{aligned}
$$


$h$ is a function of $g, h^{n}$ a function of $u_{1}, \ldots, u_{n}$. This comes from the fact that $\frac{\partial}{\partial u_{n+1}}$ commute with $\tilde{L}_{t}^{n+1}$

Therefore the two sides of (13) satisfy the same parabolic equation with second-member. We deduce that

$$
\tilde{P}_{t}^{n+1}\left[u_{n+1} \prod_{j=1}^{n} u_{j} h(.)\right](., ., 0)=\int_{0}^{t} d s \tilde{P}_{t, s}^{n}\left[f^{j_{n+1}} \alpha_{s}^{n+1} \tilde{P}_{s}^{n}\left[h \prod_{j=1}^{n} u_{j}\right]\right](., .)
$$

This is an integration by parts formula. We would like to present this formula in a more appropriate way for our object.

We consider the operator

$$
\bar{L}^{n}=L+\sum_{j=1}^{n} \frac{\partial^{2 k}}{\partial u_{j}^{2 k}}
$$

It generates a semi-group $\bar{P}_{t}^{n}$. In the sequel we will skip the problem of sign coming if $k$ is even or not. Since $\prod_{j=1}^{n} u_{j}$ is a polynomial, the Volterra expansion associated to $\tilde{P}_{s}\left[h \prod_{j=1}^{n} u_{j}\right]$ is finite and converge. We get

$$
\tilde{P}_{s}\left[h \prod_{j=1}^{n} u_{j}\right](., .)=\sum(-1)^{l} \int_{s>s_{1}>. .>s_{l}>0} I_{s_{1}, . ., s_{l}}^{l} d s_{1} . . d s_{l}
$$

where

$$
\begin{aligned}
I_{s_{1}, . ., s_{l}}^{l}=\bar{P}_{s-s_{1}}^{n}\left[\sum _ { i = 1 } ^ { n } f ^ { j _ { i } } \alpha _ { s _ { 1 } } ^ { i } \frac { \partial } { \partial u _ { i } } \left[\overline { P } _ { s _ { 1 } - s _ { 2 } } ^ { n } \left[\sum_{i=1}^{n} f^{j_{i}} \alpha_{s_{2}}^{i}\right.\right.\right. \\
\frac{\partial}{\partial u_{i}}\left[\overline { P } _ { s _ { 3 } - s _ { 2 } } ^ { n } \left[\left[\sum _ { i = 1 } ^ { n } f ^ { j _ { i } } \alpha _ { s _ { 2 } } ^ { i } \frac { \partial } { \partial u _ { i } } \left[\ldots\left[\bar{P}_{s_{l}}^{n}\left[h \prod_{j=1}^{n} u_{j}\right] . .\right](., .)\right.\right.\right.\right.
\end{aligned}
$$

Moreover

$$
\bar{P}_{s}^{n}\left[h \prod_{j=1}^{n} u_{j}\right]\left(g_{0}, .\right)=\bar{P}_{s}^{n}\left[h\left(. g_{0}\right) \prod_{j=1}^{n} u_{j}\right](e, .)
$$

such that

$$
\begin{aligned}
f^{i_{j}} \bar{P}_{s}^{n}\left[h \prod_{j=1}^{n} u_{j}\right]\left(g_{0}, .\right) & = \\
& \bar{P}_{s}^{n}\left[f^{i_{j}} h\left(. g_{0}\right) \prod_{j=1}^{n} u_{j}\right](e, .)=\bar{P}_{s}^{n}\left[f^{i_{j}} h(.) \prod_{j=1}^{n} u_{j}\right]\left(g_{0}, .\right)
\end{aligned}
$$

We remark that in (17) the series is finite and stop at $n$ because we consider a polynomial in $v_{i}$ and because $\frac{\partial}{\partial u_{i}}$ commute with $\bar{P}_{t}$. If we consider $\overline{P_{t}}\left(h_{1}(g) h_{2}(v)\right)$ it is a product of the $P_{t}\left(h_{1}\right) Q_{t}\left(h_{2}(v)\right)$ where $Q_{t}$ is generated by 
$\sum_{j=1}^{n} \frac{\partial^{2 k}}{\partial u_{j}^{2 k}}$. We deduce that in the term of the Volterra expansion of length $l$ smaller than $n$, we get $\left(P_{t-s}\left(f^{l} h(g)\right) Q_{t-s}\left(h_{1}(v)\right.\right.$ where $h_{1}(v)$ is an homogeneous polynomial with coefficient independent of $g$ of degree $n-l$.

We do the following recursion hypothesis on $l$ :

Hypothesis (l) There exists a positive real $r_{l}$ such that if $(\alpha)=\left(i_{(\alpha)}, . ., i_{(\alpha)}\right)$. is a multiindex of length smaller than 1 constituted of $|(\alpha)|$ the same element

$$
\left|P_{t}\left[f^{(\alpha)} h \prod_{i=n}^{n} u_{i}\right](g, v .)\right| \leq C t^{-r_{l}}\|h\|_{\infty}\left(1+\prod_{i=n}^{n}\left|v_{i}\right|\right)
$$

where $\|\cdot\|_{\infty}$ is the uniform norm of $h$.

It is true for $l=1$ by (01) and the next part.

If it is true for $l$, it is still true for $l+1$, by using (15) and the Volterra expansion above for $f^{(\alpha)} h$ and taking $\alpha_{s}^{n+1}=s^{r_{l}}$

By choosing suitable $\alpha_{t}^{j}$, we have according the framework of the Malliavin Calculus for any basis of the Lie algebra $f^{i}$, for any $l$

$$
\left|P_{t}\left[\sum_{i}\left(f^{i}\right)^{l} h\right]\left(g_{0}\right)\right| \leq C_{(\alpha)}\|h\|_{\infty}
$$

in order to conclude, because the operator $\sum_{i}\left(f^{i}\right)^{l}$ is an elliptic operator whose degree tends to infinity when $l \rightarrow \infty$.

\section{STUDY OF AN EXAMPLE ON THE LIN- EAR SPACE}

We give in this part a big category of examples on $\mathbb{R}^{d}$ of symmetric bounded below pseudodifferential operators which takes its origin in the theory of Poisson process $([5],[6])$.

We consider the space $C^{\infty}\left(\mathbb{R}^{d}\right)$ of smooth functions $h$ with bounded derivatives at each order.

We introduce a smooth function from $\mathbb{R}^{d} \times \mathbb{R}^{d}$ into $\mathbb{R}(x, y) \rightarrow g(x, y)$ which is equals to 0 if $|y|>C>0$ and with bounded derivatives at each order. This allows us to introduce the integro-differential operator on $C^{\infty}\left(\mathbb{R}^{d}\right)$ :

$$
\begin{aligned}
\operatorname{Lh}(x)=(-1)^{l+1} \int_{\mathbb{R}^{d}}(h(x+y)-h(x) & \\
& \left.\quad-\sum_{i=1}^{2 l} 1 / i !<y^{\otimes i}, h^{(i)}(x)\right) g(x, y)|y|^{-(2 l+d+\alpha)} d y
\end{aligned}
$$

for $\alpha \in]-1,0[$.

We do the following hypothesis: for all $x \in \mathbb{R}^{d}, h(x, 0)>C>0$. 
In such a case, we have shown ([8], [9]) that $L$ is a pseudodifferential elliptic operator with symbol

$$
\begin{aligned}
a(x, \xi)=(-1)^{l+1} \int_{\mathbb{R}^{d}}(\exp [\sqrt{-1}<y, \xi>]- & \\
& \left.\sum_{i=1}^{2 l} 1 / i !(\sqrt{-1}<y, \xi>)^{i}\right) g(x, y)|y|^{-(2 l+d+\alpha)} d y
\end{aligned}
$$

$L$ is elliptic and satisfies to Garding assumption (11) with $m^{\prime} \rightarrow \infty$ when $l \rightarrow \infty$. We produce a large class of examples of such operators which are moreover symmetric in $L^{2}(d x)$.

Let be $X_{j}(x), j=1, . ., d$ be some vector firlds without divergence, with bounded derivatives of each order and which are uniformly in $x$ in $\mathbb{R}^{d}$ a basis of $\mathbb{R}^{d}$.

Let $\phi_{t}(y)(x)$ be the dynamical system generated by the vector field $X(y, x)=$ $\sum_{j=1}^{d} y_{j} X_{j}(x) ; \phi_{0}(y)(x)=x$ and

$$
d \phi_{t}(y)(x)=X\left(y, \phi_{t}(y)\right) d t
$$

We suppose $g(x, y)=g(y)=g(-y)$ with a small support.

We introduce the operator

$$
\begin{aligned}
L_{1} h(x)=(-1)^{l+1} \int_{\mathbb{R}^{d}}( & h\left(\phi_{1}(y)(x)\right)-h(x)- \\
& \left.\sum_{i=1}^{l} 1 /(2 i !)(X(y, x))^{(2 i)} h(x)\right) g(y)|y|^{-(2 l+d+\alpha)} d y
\end{aligned}
$$

In the previous formula, the vector field $X(y, x)$ is considered as a one order differential operator in $x$.

Lemma 2 Under the symmetry condition on $g, L_{1}$ is symmetric and is defined on $C^{\infty}\left(\mathbb{R}^{d}\right)$.

Proof: The fact that $L_{1}$ is defined on $C^{\infty}\left(\mathbb{R}^{d}\right)$ comes from the fact that the asymptotic expansion of $y \rightarrow h\left(\phi_{1}(y)(x)\right.$ near 0 is

$$
h(x)+\sum_{i=1}^{2 l} 1 / i ! X(y, x)^{(i)} h(x)
$$

and from the fact that $g(y)=g(-y)$ such that only even integers remain in the sum (23).

The fact that $L_{1}$ is symmetric comes from two fact: the vector field $X(y, x)$ is divergence free such that

$$
\int_{\mathbb{R}^{d}} h_{1}(x) X(y, x)^{(2 i)} h_{2}(x) d x=\quad=\quad \int_{\mathbb{R}^{d}} h_{2}(x) X(y, x)^{(2 i)} h_{1}(x) d x
$$


by integrating by part. Moreover $x \rightarrow \phi_{1}(y)(x)$ preserves the Lebesgue measure such that

$$
\int_{\mathbb{R}^{d}} h_{1}(x) h_{2}\left(\phi_{1}(y)(x)\right) d x=\int_{\mathbb{R}^{d}} h_{1}\left(\phi_{1}(-y)(x)\right) h_{2}(x) d x
$$

and the result arises from the equality $g(y)=g(-y)$.

$\diamond$

Theorem $3 L_{1}$ is an operator of the type (23) which is symmetric bounded below.

Proof: It remains only to show that $L_{1}$ is an operator of the type (23). For that we remark that the map

$$
y \rightarrow \phi_{1}(y)(x)-x
$$

is a local diffeomorhism at every point $y$ and a local diffeomorphism of a neighborhood of 0 in $\mathbb{R}^{d}$ onto a neighborhood of 0 in $\mathbb{R}^{d}$.

$\diamond$

Remark: Let us give some heuristic explanation which explain this part. Let us consider a formal path measure $d Q$ on a "space" of paths $y_{t}$ with jumps starting from 0 which represents the semi-group $P_{t}$ associated to the operator

$$
\begin{aligned}
\operatorname{Lh}(x)=(-1)^{l+1} \int_{\mathbb{R}^{d}}( & h(x+y)-h(x)- \\
& \left.\sum_{i=1}^{l} 1 /(2 i !)<y^{\otimes 2 i}, h^{(2 i)}(x)>\right) g(y)|y|^{-(2 l+d+\alpha)} d y
\end{aligned}
$$

such that formally

$$
P_{t} h(x)=\int h\left(y_{t}+x\right) " d Q(y .) "
$$

We consider the "formal stochastic differential with jumps" whose solution (starting from $x$ ) $y_{1, t}(x)$ satisfies

$$
\Delta y_{1, t}(x)=\phi_{1}\left(\left(\Delta y_{t}\right)\right)\left(y_{1, t-}(x)\right)-y_{1, t-}(x)
$$

where $y_{t-}=\lim _{s \rightarrow t-} y_{s}$ and $\Delta y_{t}=y_{t}-y_{t-}$. We should get

$$
P_{1, t} h(x)=\int f\left(y_{1, t}(x) " d Q(y .) "\right.
$$

Moreover, a lot of compensation should appear in the formal equation giving $y_{1, t}$. We refer to [5] in the case where the path integrals are rigorously defined (In such a case only one compensation appears!) 


\section{References}

[1] J.M. Bismut, "Calcul des variations stochastiques et processus de sauts", Z. Wahr. Verw. Gebiete, 63, pp. 147-235, 1983.

[2] J. Chazarain, A. Piriou, Introduction a la théorie des équations aux dérivées partielles linéaires. Paris, France: Gauthier-Villars, 1981.

[3] L. Hoermander, The analysis of linear partial operators III. Berlin, Germany: Springer, 1984.

[4] L. Hoermander, The analysis of linear partial operators IV. Berlin, Germany: Springer, 1984.

[5] Ishikawa Y., Stochastic Calculus of variations for jump processes, Basel, Schweiz: de Gruyter, 2012.

[6] R. Léandre, "Extension du théoreme de Hoermander a divers processus de sauts", PHD Thesis, Université de Besançon, France (1984)

[7] R. Léandre, "Stochastic analysis for a non-markovian generator: an introduction", Russian Journal of Mathematical Physics, 22, pp 39-52, 2015.

[8] R. Léandre,"Large deviation estimates for a non-Markovian generator of Lévy type of big order". 4th Int. Conf. Math. Modern. Phys. Sciences, Journal of physics: conference series, 633, 012085, 2015 (E. Vagenas and al esds).

[9] R. Léandre," A Class of non-Markovian pseudo-differential operators of lévy type". Pseudo-differential operators: groups, geometry and applications. Birkhauser (2017), 149-159 (M.W. Wong and al eds).

[10] R. Léandre, "Perturbation of the Malliavin Calculus of Bismut type of large order". Phy Mathematical aspects of symmetries Springer (2017), 221-225. (J.P. Gazeau and al eds).

[11] R. Léandre, "Malliavin Calculus of Bismut type for an operator of order four on a Lie group". Journal of Pseudo-differential operators and applications, 8, pp 419-430, 2019.

[12] R. Léandre, "Bismut's way of the Malliavin Calculus of large order generators on a Lie group".6th Int. Eur. Conf. Math. Sciences and Applications 1926 (2018), 020026A.I.P. Proceedings (M. Tosun and al eds).

[13] R. Léandre,"Bismut's way of the Malliavin Calculus for non markovian semi-groups: an introduction" to appear in Analysis of pseudo-differential operators. M.W. Wong and al eds.

[14] R.T. Seeley, "Complex powers of an elliptic operator" In Singular integralsProc. Symp. Pure. Mathematics. Providence, U.S.A. A.M.S. (1966), 288-307. 
[15] M. Taylor, Partial differential equations II. Qualitative studies of linear equations, Heidelberg, Germany: Springer, 1997. 